\title{
Contributors to diffusion impairment in HIV-infected persons
}

\author{
Matthew R. Gingo ${ }^{1}$, Jiayan He², Catherine Wittman ${ }^{1}$, Carl Fuhrman ${ }^{3}$, \\ Joseph K. Leader ${ }^{3}$, Cathy Kessinger ${ }^{1}$, Lorrie Lucht', William A. Slivka', \\ Yingze Zhang ${ }^{1}$, Deborah K. McMahon ${ }^{1}$, Frank C. Sciurba ${ }^{1}$ and Alison Morris ${ }^{1,4}$
}

\author{
Affiliations: \\ 'Dept of Medicine, University of Pittsburgh, Pittsburgh, PA, \\ ${ }^{2}$ Dept of Biostatistics, Graduate School of Public Health, University of Pittsburgh, Pittsburgh, PA, \\ ${ }^{3}$ Dept of Radiology, University of Pittsburgh, Pittsburgh, PA, and \\ ${ }^{4}$ Dept of Immunology, University of Pittsburgh, Pittsburgh, PA, USA.
}

\section{Correspondence:}

A. Morris, 3459 Fifth Avenue, 628 NW, Pittsburgh, PA 15213, USA.

E-mail: morrisađupmc.edu

ABSTRACT Abnormal diffusing capacity is common in HIV-infected individuals, including never smokers. Aetiologies for diffusing capacity impairment in HIV are not understood, particularly in those without a history of cigarette smoking.

Our study was a cross-sectional analysis of 158 HIV-infected individuals without acute respiratory symptoms or infection with the aim to determine associations between a diffusing capacity of the lung for carbon monoxide (DLCO) \% predicted and participant demographics, pulmonary spirometric measures (forced expiratory volume in $1 \mathrm{~s}$ (FEV1) and FEV1/forced vital capacity), radiographic emphysema (fraction of lung voxels $<-950$ Hounsfield units), pulmonary vascular/cardiovascular disease (echocardiographic tricuspid regurgitant jet velocity, $\mathrm{N}$-terminal pro-brain natriuretic peptide) and airway inflammation (induced sputum cell counts), stratified by history of smoking.

The mean DLCO was $65.9 \%$ predicted, and $55(34.8 \%)$ participants had a significantly reduced DLCO $(<60 \%$ predicted). Lower DLCO $\%$ predicted in ever-smokers was associated with lower postbronchodilator FEV1 \% predicted $(\mathrm{p}<0.001)$ and greater radiographic emphysema $(\mathrm{p}=0.001)$. In neversmokers, mean \pm SD DLCO was $72.7 \pm 13.4 \%$ predicted, and DLCO correlated with post-bronchodilator FEV1 $(\mathrm{p}=0.02)$, sputum neutrophils $(\mathrm{p}=0.03)$ and sputum lymphocytes $(\mathrm{p}=0.009)$, but not radiographic emphysema.

Airway obstruction, emphysema and inflammation influence DLCO in HIV. Never-smokers may have a unique phenotype of diffusing capacity impairment. The interaction of multiple factors may account for the pervasive nature of diffusing capacity impairment in HIV infection.

@ERSpublications

Diffusing capacity impairment in HIV infection is multifactorial, and causes may vary between smokers and never smokers http://ow.ly/qag5I

Received: Oct 042012 | Accepted after revision: Jan 312013 | First published online: Feb 212013

Support statement: Sources of support were as follows: National Institutes of Health (NIH) T32 HL007563 and K23 HL108697 (to M.R. Gingo); P50 HL084948 and N01 HR46163R01 (to F.C. Sciurba); R01 HL083461, HL083461S and HL090339 (A. Morris); and the University of Pittsburgh CTSI (UL1 RR024153).

Conflict of interest: Disclosures can be found alongside the online version of this article at www.erj.ersjournals.com 


\section{Introduction}

Lung disease is an important cause of morbidity and mortality in the HIV-infected population even in the current era of combination antiretroviral therapy (ART). A greater incidence of several noninfectious lung diseases has been found in HIV-infected persons compared with HIV-uninfected persons [1], and death from obstructive lung disease has increased in the HIV-infected population since the introduction of ART [2]. Abnormal diffusing capacity and an accelerated form of emphysema were associated with HIV infection prior to ART [3-6]. A recent study showed that abnormal diffusing capacity remains extremely common with $>64 \%$ of HIV-infected persons having an impaired diffusing capacity of the lung for carbon monoxide (DLCO) $(<80 \%$ predicted). Diffusing capacity impairment is not limited to smokers with HIV as $>47 \%$ of never-smokers have been reported to have a DLCO $<80 \%$ pred [7]. Additionally, diffusing capacity impairment in the HIV-uninfected population has been associated with increased mortality [8], highlighting the importance of this abnormality in lung function.

DLCO can be decreased by multiple mechanisms, including parenchymal destruction, interstitial lung disease, loss of alveolar surface or primary pulmonary vascular processes. The contributors to decreased diffusing capacity in HIV are not well known, but studies prior to ART found the majority of HIV-infected individuals had impairments in DLCO related to advanced HIV, infections and emphysema [3-6]. One study from a pre-ART cohort of participants without AIDS-defining lung disease demonstrated impairment in diffusing capacity was related to a decrease in capillary blood volume and not an increase in the membrane component of gas diffusion, suggesting emphysema or pulmonary vascular disease to be the significant contributors [4]. Although recent studies have found that abnormal DLCO remains common in HIV infection in the post-ART era, specific contributors to this abnormality have not been examined, and associations in nonsmokers who may represent a distinct phenotype have not been specifically investigated. Understanding causes of diffusion impairment in HIV might lead to the development of novel therapies.

We investigated contributors to impaired diffusing capacity in an HIV-infected cohort. Factors examined included mechanical lung function, computed tomography (CT)-assessed emphysema, echocardiographic pulmonary hypertension, markers of cardiac strain and lung inflammation. We also investigated the same relationships with impaired diffusing capacity in the subset of participants who had never smoked.

\section{Methods}

\section{Participants}

Participants were $158 \mathrm{HIV}$-infected outpatients and were a subset of an established cohort recruited from the University of Pittsburgh HIV/AIDS clinic (Pittsburgh, PA, USA) who had a study visit between February 2009 and August 2011 [7]. All participants signed written informed consent, and the University of Pittsburgh Institutional Review Board approved the protocol. Standardised questionnaires were used to obtain demographic and clinical data including smoke exposure and smoking history, any occupational exposures to vapours, gases, dusts or fumes, and prior pneumonia. Medical record review was used to obtain $\mathrm{CD}^{+}$T-lymphocyte count and plasma HIV RNA levels within the past 6 months.

\section{Testing procedures}

Participants performed spirometry and single-breath DLCO measurement according to the American Thoracic Society/European Respiratory Society guidelines [9, 10]. Race-adjusted predicted values for spirometry were determined using Hankinson formulas and for DLCO using Neas formulas adjusted for carboxyhaemoglobin and haemoglobin concentrations $[9,11,12]$. Standardised noncontrast CT scans of the entire thorax at end-inspiration were obtained in individuals who had less than $\sim 10$ rad exposure to radiation in the prior year. The percentage of lung voxels associated with emphysema defined as voxels below -950 Hounsfield units was calculated [13]. Scans were reviewed independently without knowledge of the participant's lung function or clinical characteristics by two pulmonologists (M.R. Gingo and C. Wittman) and a radiologist (C. Fuhrman) to determine the presence of interstitial lung disease or fibrosis defined by the presence of diffuse, peripheral or subpleural patchy ground-glass opacities, reticular opacities, honeycomb changes or signs of volume loss (traction bronchiectasis and displaced fissure) [14]. Echocardiography was performed to determine peak tricuspid regurgitant jet velocity (TRV) [15], left ventricular (LV) ejection fraction, LV hypertrophy and diastolic dysfunction. A TRV $\geqslant 3.0 \mathrm{~m} \cdot \mathrm{s}^{-1}$ was defined as elevated [16]. Echocardiograms were added to the study protocol as of July 1, 2009 and were available in 126 individuals. Percentages of neutrophils, lymphocytes and eosinophils were determined from sputum induced via inhalation of nebulised 3\% saline [17]. Sputum samples were considered suitable for analysis if they contained $<30 \%$ squamous cells $(n=128)$. Plasma $N$-terminal pro-brain natriuretic peptide (NT-proBNP) was measured in participants who had a modification of diet in renal disease [18] estimated glomerular filtration rate $\geqslant 60 \mathrm{~mL} \cdot \mathrm{min}^{-1}$ per $1.73 \mathrm{~m}^{2}(\mathrm{n}=143)$ [19]. CT scans and echocardiograms were administered by trained clinical technicians. Pulmonary function tests were administered by 
either of two trained research nurses. Sputum analysis and NT-proBNP measurement were performed in a research laboratory.

\section{Statistical analysis}

Because it is likely that causes of diffusion impairment are different in those who had ever-smoked and never-smokers, analysis was stratified by smoking status. The dependent variable DLCO \% pred was analysed as a dichotomous variable, identifying those with more significantly impaired diffusing capacity $(\leqslant 60 \%$ pred) versus $>60 \%$, and also as a continuous variable. This cut-off was chosen because it is used clinically to define moderate impairment of DLCO and we were interested in a more severe phenotype. Participant characteristics were summarised and compared between participants with a DLCO $>60 \%$ pred and $\leqslant 60 \%$ pred using t-tests, Wilcoxon rank-sum, Chi-squared or Fisher's exact tests, where appropriate, with a p-value $<0.05$ considered to be significant. Variables of interest associated with DLCO were assessed under four categories: 1) measures of obstructive lung disease (forced expiratory volume in $1 \mathrm{~s}$ (FEV1) \% pred, forced vital capacity (FVC) \% pred, FEV1/FVC, and per cent of radiographic emphysema); 2) interstitial lung disease (presence of interstitial changes on CT scan); 3) pulmonary vascular/cardiovascular disease (TRV, elevated TRV, NT-proBNP and LV function); and 4) airway inflammation (induced sputum cell counts). To approximate normality, NT-proBNP was transformed using the natural logarithm and sputum lymphocyte counts using the square root. Sputum eosinophils were dichotomised for analysis to detectable versus absent. Associations between variables of interest and DLCO \% pred were determined using simple linear regression.

Two linear regression models, one for ever-smokers and another for never-smokers, were created to determine independent associations between DLCO \% pred and participant demographics, obstructive lung disease, pulmonary vascular disease and inflammation. Variables were selected for the model that had univariate associations of $\mathrm{p}<0.1$. Models were selected using stepwise regression [20]. The multivariable models were assessed for excess colinearity by checking variance inflation factors [21]. The effect of secondhand smoke exposure was assessed in the multivariate models and no significant confounding was noted.

\section{Results}

158 participants completed pulmonary function testing, 125 who had ever-smoked and 33 who had neversmoked. The majority of participants $(71.3 \%)$ had undetectable plasma HIV RNA levels, and CD4 counts at the most recent assessment within 6 months prior to study visit were generally high (median CD4 count 561 cells $\cdot \mu \mathrm{L}^{-1}$, range $24-2094$ cells $\cdot \mu \mathrm{L}^{-1}$ ). The mean \pm SD DLCO was $64.1 \pm 15.0 \%$ pred in ever-smokers and $72.7 \pm 13.4 \%$ pred in never-smokers (table 1). $84 \%$ of participants had a DLCO $<80 \%$ pred. $47(37.6 \%)$ smokers had a more significantly reduced DLCO ( $\leqslant 60 \%$ pred) and even eight $(24.2 \%)$ never-smokers had a DLCO $\leqslant 60 \%$ pred (fig. 1). Among smokers, those with a reduced DLCO had lower body mass index, smoked more pack-years and were more likely to have used cocaine (table 1). In 125 participants with data collected on secondhand smoke exposure, secondhand smoke exposure during childhood was more common in ever-smokers with significantly reduced DLCO.

\section{Measures of obstructive lung disease}

Diffusing capacity was associated with several measures of obstructive lung disease. In ever-smokers, a lower DLCO \% pred was associated with lower post-bronchodilator FEV1, FVC and FEV1/FVC (table 2), but in never-smokers, lower DLCO \% pred was only associated with FEV1 and FVC. In 117 participants who had CT scans, a lower DLCO \% pred was seen with a higher fraction of lung voxels $<-950$ Hounsfield units in ever-smokers but not never-smokers (fig. 2).

\section{Measures of interstitial lung disease}

None of the CT scans reviewed were rated as positive for the presence of interstitial lung disease or fibrosis.

\section{Measures of pulmonary vascular/cardiovascular disease}

Lower DLCO \% pred was seen with a higher TRV and higher NT-proBNP levels in ever-smokers. 10 (7.9\%) participants, all ever-smokers, had echocardiograms with an abnormal TRV $\left(\geqslant 3.0 \mathrm{~m} \cdot \mathrm{s}^{-1}\right)$ and an abnormal TRV was associated with a lower DLCO \% pred. Measures of LV function were not associated with DLCO in ever-smokers, but lower LV ejection fraction was associated with lower DLCO \% pred in neversmokers (table 2).

\section{Measures of airway inflammation}

The mean \pm SD percentage of neutrophils in 128 participants with adequately induced sputum was $50.5 \pm 19.8 \%$. None of the sputum cell counts were associated with DLCO in ever-smokers, but higher 


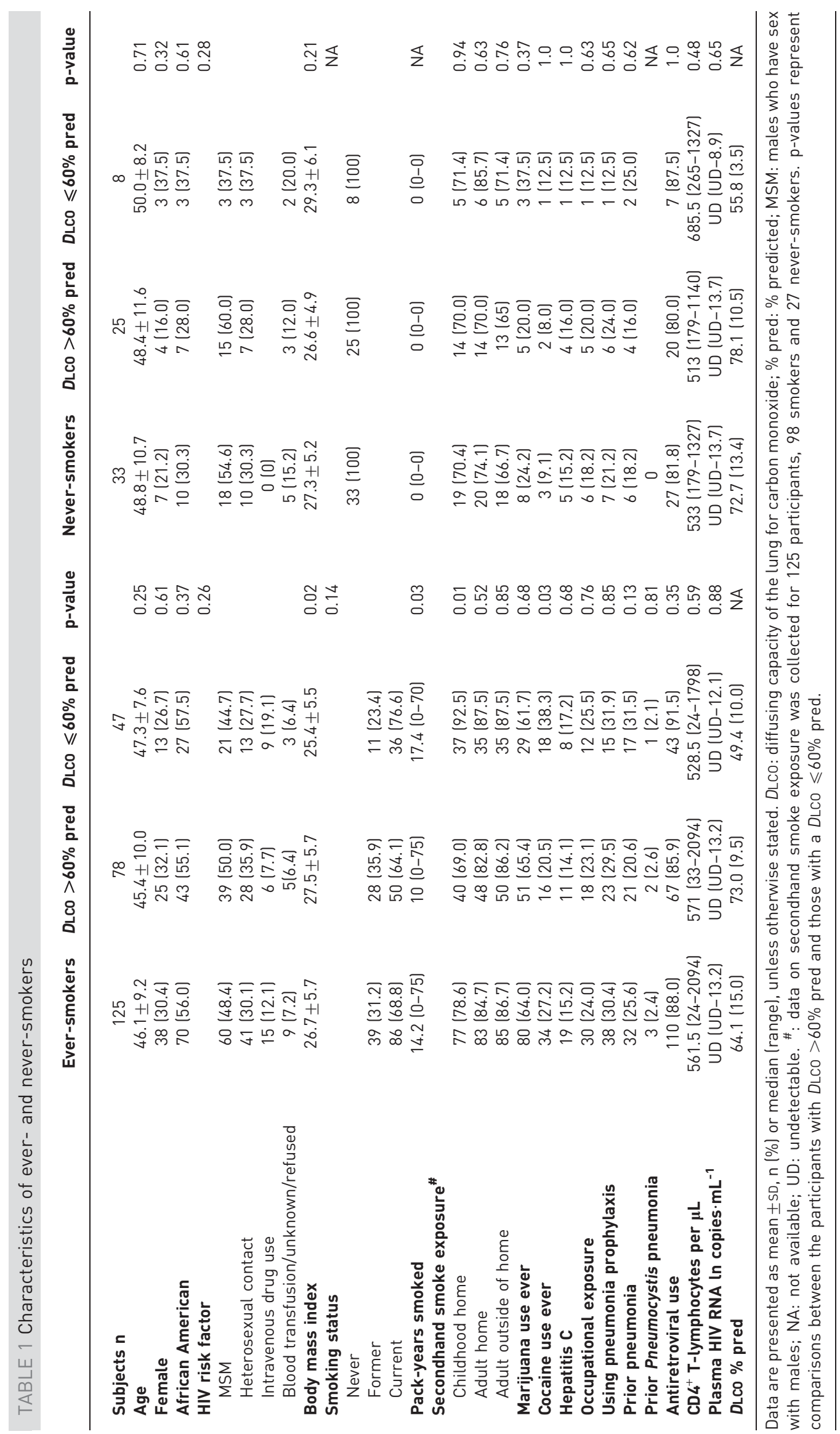


FIGURE 1 Distribution of diffusing capacity of the lung for carbon monoxide (DLCO) \% predicted (\% pred). Dashed line represents DLCO 60\% pred. Solid line represents the mean.

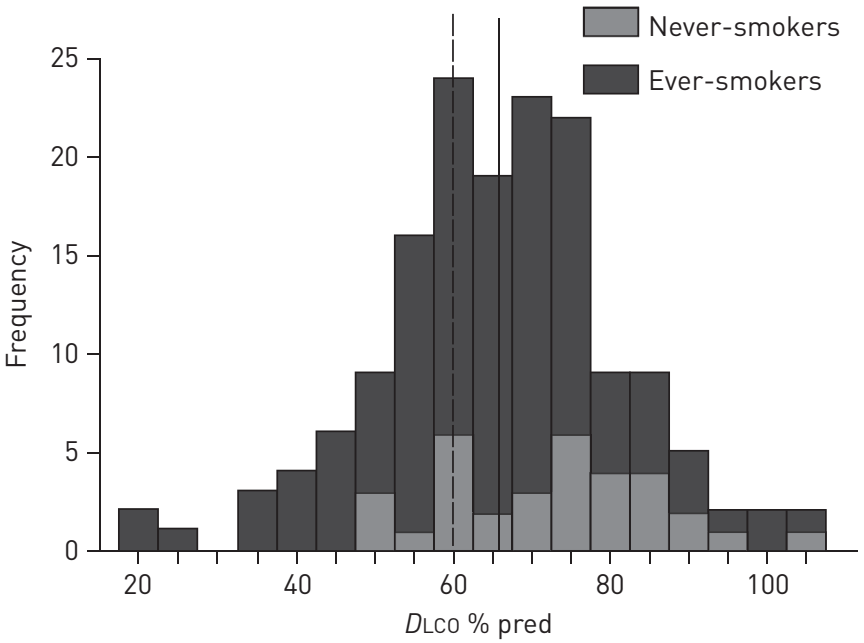

sputum neutrophil percentage was associated with a lower DLCO \% pred in never-smokers (fig. 2). The median (range) lymphocyte percentage was $0.7 \%(0-9.0 \%)$, and lower sputum lymphocyte percentages were associated with lower DLCO in never-smokers.

While the associations between DLCO and post-bronchodilator FEV1 \% pred, TRV and sputum neutrophils (figs 2a, b and c) were similar in smokers and never-smokers, the associations appeared different between smokers and never-smokers for post-bronchodilator FEV1/FVC and radiographic emphysema (figs $2 \mathrm{~d}$ and $\mathrm{e}$ ).

\section{Multivariable regression}

Regression models were evaluated to determine specific factors independently associated with impaired diffusion in ever-smokers and never-smokers separately (table 3). In ever-smokers, the final model showed that lower post-bronchodilator FEV1 \% pred and greater percentage emphysema measured by the fraction

TABLE 2 Univariate associations of markers of airway obstruction, pulmonary hypertension and airway inflammation with diffusing capacity of the lung for carbon monoxide (DLCO) \% predicted ( $\%$ pred)

\section{DLco \% pred}

\begin{tabular}{|c|c|c|c|}
\hline \multicolumn{2}{|c|}{ Ever-smokers } & \multicolumn{2}{|c|}{ Never-smokers } \\
\hline$\beta$-coefficient & p-value & $\beta$-coefficient & p-value \\
\hline 0.4245 & $<0.001$ & 0.3679 & 0.001 \\
\hline 0.3255 & $<0.001$ & 0.4743 & $<0.001$ \\
\hline 0.6748 & $<0.001$ & 0.2084 & 0.62 \\
\hline-0.0564 & $<0.001$ & 0.0191 & 0.52 \\
\hline-0.1123 & 0.02 & -0.0827 & 0.41 \\
\hline-0.1572 & 0.002 & & \\
\hline 0.0024 & 0.512 & 0.0205 & 0.02 \\
\hline-0.0290 & 0.46 & 0.0971 & 0.13 \\
\hline-0.0182 & 0.65 & -0.0113 & 0.91 \\
\hline-0.0153 & 0.14 & 0.0036 & 0.91 \\
\hline-0.1025 & 0.17 & -0.2684 & 0.03 \\
\hline-0.0321 & 0.27 & -0.0105 & 0.86 \\
\hline 0.0546 & 0.75 & 1.3000 & 0.003 \\
\hline
\end{tabular}

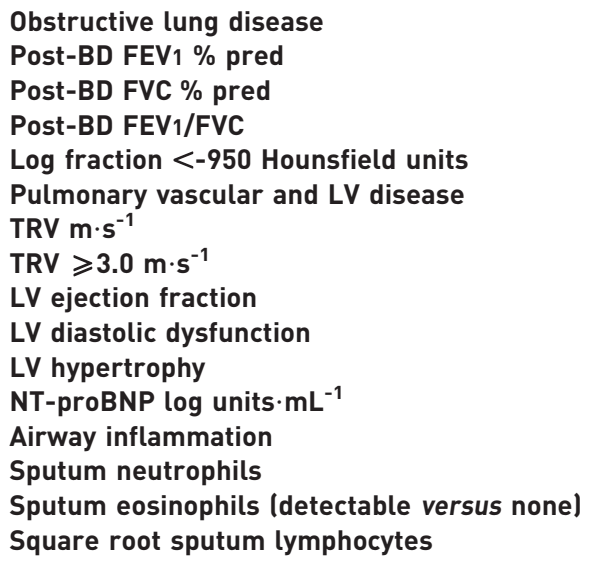

BD: bronchodilator; FEV1: forced expiratory volume in $1 \mathrm{~s}$; FVC: forced vital capacity; LV: left ventricle; TRV: tricuspid regurgitant jet velocity; NTproBNP: N-terminal pro-brain natriuretic peptide. 

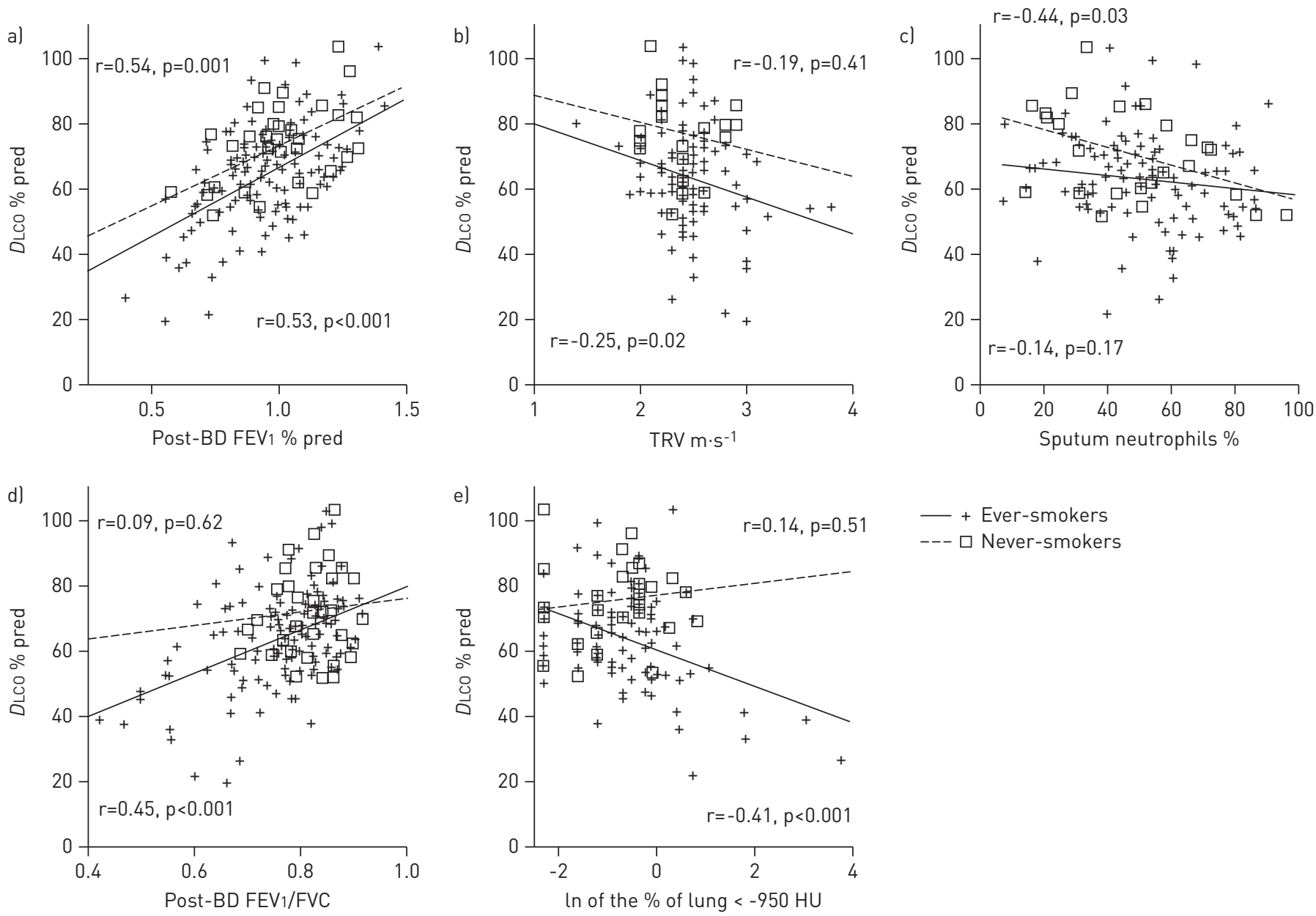

FIGURE 2 Scatter plots and regression lines for diffusing capacity of the lung for carbon monoxide (DLCO) \% predicted (\% pred) by a) post-bronchodilator (post-BD) forced expiratory volume in $1 \mathrm{~s}$ (FEV1) \% pred, b) tricuspid regurgitant jet velocity (TRV), c) sputum neutrophil percentage, d) post-BD FEV1/forced vital capacity (FVC) and e) natural logarithm (ln) of the fraction of lung <-950 Hounsfield units (HU) for ever-smokers (+ and solid lines) and never-smokers (open squares and dashed lines). Pearson correlation coefficients ( $r$ ) and significance values (p) are shown nearest to the regression lines of smokers (bottom) and never-smokers (top) for correlations between the independent variable in each graph and DLCO \% pred.

of lung <-950 Hounsfield units were independently associated with worse DLCO \% pred. In contrast with this, in never-smokers, lower DLCO \% pred was associated with lower post-bronchodilator FVC \% pred, a greater percentage of neutrophils in sputum, and a lower percentage of lymphocytes in sputum.

TABLE 3 Multivariable regression models showing independent associations for diffusing capacity of the lung for carbon monoxide (DLCO) \% predicted (\% pred) in ever-smokers and never-smokers

\begin{tabular}{|c|c|c|c|c|}
\hline & \multicolumn{2}{|c|}{ Ever-smokers ${ }^{\#}$} & \multicolumn{2}{|c|}{ Never-smokers } \\
\hline & $\beta$-coefficient & p-value & $\beta$-coefficient & p-value \\
\hline Post-BD FEV1 \% pred & 0.3940 & $<0.001$ & & \\
\hline Post-BD FVC \% pred & & & 0.3323 & 0.02 \\
\hline Log fraction $<-950$ Hounsfield units & -0.0423 & 0.001 & & \\
\hline$\%$ Sputum neutrophils & & & -0.1967 & 0.03 \\
\hline$\%$ Sputum lymphocytes square root & & & 0.9407 & 0.009 \\
\hline
\end{tabular}

FEV1: forced expiratory volume in $1 \mathrm{~s}$; BD: bronchodilator; FVC: forced vital capacity. ": body mass index, pack-years smoking and cocaine use were considered for model construction. 


\section{Discussion}

In an HIV-infected cohort, we found that impaired diffusing capacity is common, even in never-smokers, and may result from different mechanisms in smokers and nonsmokers. Overall, in smokers, impaired diffusing capacity was associated with measures related to obstruction and emphysema (lower FEV1 \% pred and greater radiographic emphysema) and, in never-smokers, diffusing capacity impairment was associated with FVC and airway inflammation, but not lower FEV1/FVC or radiographic emphysema.

Degree of diffusing capacity impairment in this cohort is comparable to findings in prior studies of diffusing capacity in HIV-infected persons [3-6]. We found a mean DLCO of 65.9\% pred, and most previous studies find low DLCO in HIV-infected individuals. In our cohort, $34.3 \%$ had a DLCO $<60 \%$ pred and $84.8 \%$ had a DLCO $<80 \%$ pred, while prior studies found $55 \%$ had a DLCO $<80 \%$ pred $[3,6]$ or $25 \%$ had a DLCO $<72 \%$ pred [4].

The pathogenesis for diffusing capacity impairment in the HIV-infected population is not completely understood. Early in the HIV epidemic, it was thought to be primarily due to HIV-related inflammation or infection [3, 5], and diffusing capacity seemed to worsen with HIV progression [3]. Prior work was performed before widespread use of effective ART, and many participants had acute pulmonary processes [3-6]. In contrast, $87 \%$ of participants in the current study were using ART and the majority had CD4 $4^{+}$ T-lymphocyte cell counts $>500$ cells $\cdot \mu \mathrm{L}^{-1}$ and undetectable viral loads $\left(<50\right.$ copies $\cdot \mathrm{mL}^{-1}$ by ultrasensitive assays). None of the participants had acute pulmonary infections, and these factors were not associated with DLCO impairment in the cohort.

The impairment of diffusing capacity in HIV in the pre-ART era was also related to a decrease in the capillary blood volume in the lung and an accelerated form of emphysema [4]. The current study supports emphysema contributing to diffusing capacity impairment in smokers; however, the prevalence of diffusing capacity impairment in never-smokers suggests diffusing capacity impairment is not entirely smoking related.

Prior work has also demonstrated reductions in diffusing capacity in HIV related to lung infection or inflammation [3,5]. In the HIV-uninfected population, increased sputum neutrophils are a hallmark of chronic obstructive pulmonary disease and correlate with disease severity [22, 23]. In our cohort, airway inflammatory markers were associated with reduced diffusing capacity only in never-smokers. However, because we do not see an association between measures of emphysema and diffusing capacity impairment in never-smokers, other mechanisms linking increased neutrophils and diffusing capacity impairment may also be important.

Pulmonary vascular disease (or pulmonary arterial hypertension) is more prevalent in HIV-infected persons $[16,24]$ and could also contribute to impairment in diffusing capacity. We found that elevated pulmonary artery pressures (as measured by increased TRV on echocardiography) correlated with decreases in diffusing capacity. We have previously shown that lower diffusing capacity is associated with elevated TRV, but our analysis demonstrates that there is an independent contribution of pulmonary artery pressures to diffusing capacity [25]. It is also possible that chronic obstructive pulmonary disease contributed to the increased pulmonary artery pressures seen, and longitudinal or animal studies will be helpful in examining cause and effect of these abnormalities.

Finally, it is possible that there are extrapulmonary causes for reduced diffusing capacity. We corrected for anaemia, but hyperglycaemia is underappreciated in HIV-infected persons, and chronic hyperglycaemia can reduce diffusing capacity $[26,27]$. We did not have measures of haemoglobin A1C to assess chronic glucose levels. Cardiac function might also impact diffusing capacity, but there was no correlation between LV function (both systolic or diastolic) or hypertrophy and DLCO except in the never-smokers, although this association was quite modest. Cardiac strain, as indicated by NT-proBNP levels, was associated with DLCO abnormalities and may reflect right heart strain in smokers given the association of elevated pulmonary artery pressures with DLCO or left heart strain in nonsmokers, given the association with LV function.

The degree of diffusing capacity impairment in never-smokers is striking (more than one-quarter have a DLCO $<60 \%$ pred) and may indicate causes for diffusing capacity impairment that are independent of smoking. Although a small sample, we found that impaired diffusing capacity in never-smokers was associated with reduced FVC, but not a reduced FEV1/FVC or radiographic emphysema. Because decreased FEV1/FVC ratio and radiographic emphysema are suggestive of an emphysema phenotype [28], emphysema may play a large role in diffusing capacity impairment in smokers, but in never-smokers, diffusing capacity impairment may be driven by a process other than emphysema or a process that might later develop into emphysema. We speculate that a component of restrictive lung physiology and inflammation or immune reconstitution may be in part responsible for impaired diffusing capacity in HIV-infected individuals. These mechanisms may be overshadowed by smoking, but still could be important contributors to diffusing capacity impairment. 
Taken together, these data suggest diffusing capacity impairment in HIV infection is multifactorial and not solely the result of accelerated chronic obstructive pulmonary disease that has been reported. The associations of diffusing capacity impairment with measures of airflow obstruction, pulmonary vascular disease and airway inflammation indicate that several pathological processes contribute to the reduction in diffusing capacity commonly seen in HIV-infected individuals. Various pathways may be contributing to these pathologies, such as smoking leading to airway obstruction and emphysema, HIV-viral proteins contributing to pulmonary vascular disease [29] or immune reconstitution leading to lung inflammation [30]. Vascular dysfunction may also link smoking and emphysema [31]. However, it is possible that these are tied together by a single underlying mechanism, such as immune activation related to HIV infection [32]. Chronic immune stimulation is believed to cause premature ageing and immune senescence in HIVinfected persons [33], and has been implicated in both emphysema [34] and pulmonary vascular disease in the HIV-uninfected population [35]. Understanding individual factors important in diffusing capacity abnormalities in this population could have direct implications for guiding appropriate diagnostic evaluation in HIV-infected patients with respiratory symptoms and an abnormal diffusing capacity, in turn influencing the treatment modalities pursued.

This study has several limitations. It is a single-centre study with a high prevalence of smoking and drug use in the cohort and may not be applicable to the entire HIV population, although smoking and drug use are quite common in HIV-infected individuals [36]. We did not evaluate other diseases that could affect diffusing capacity and have been reported more frequently in HIV, such as primary pulmonary hypertension or lymphocytic interstitial pneumonia [1]. However, these diseases are still rare, even in HIV infection, and they would be unlikely to be responsible for the great degree of impaired diffusing capacity seen. We have cross-sectional data on $\mathrm{CD}^{+}$T-lymphocyte cell and plasma HIV RNA levels, but peak and nadir levels or changes over time may be important factors in the development of impaired diffusing capacity. Pre-therapy HIV RNA levels, HIV-specific immune responses, host genetic factors or immune activation/chronic inflammation may also be important. We have a relatively small sample of neversmokers, which may limit the power to detect meaningful associations. Additionally, the degree and magnitude of secondhand smoke exposure is difficult to quantify and, with $>70 \%$ of never-smokers reporting some secondhand smoke exposure, it raises the question as to whether they are truly without smoke-related injury.

In conclusion, abnormal diffusing capacity is common in HIV-infected persons, even in nonsmokers. While smoking and illicit drug use are associated with abnormal diffusing capacity, independent contributions of obstructive lung disease, primary pulmonary vascular disease and inflammation suggest either a multifactorial cause of diffusing capacity impairment in HIV-infected individuals or a common, underlying pathway linking these processes. Given that reduced DLCO also predicts mortality [8], the driving forces resulting in accelerated emphysema, pulmonary vascular disease and inflammation in HIV infection are important to understand in order to ameliorate this significant impairment in lung function.

\section{References}

Crothers K, Huang L, Goulet JL, et al. HIV infection and risk for incident pulmonary diseases in the combination antiretroviral therapy era. Am J Respir Crit Care Med 2011; 183: 388-395.

2 Louie JK, Hsu LC, Osmond DH, et al. Trends in causes of death among persons with acquired immunodeficiency syndrome in the era of highly active antiretroviral therapy, San Francisco, 1994-1998. J Infect Dis 2002; 186: 1023-1027. Nieman RB, Fleming J, Coker RJ, et al. Reduced carbon monoxide transfer factor (TLCO) in human immunodeficiency virus type I (HIV-I) infection as a predictor for faster progression to AIDS. Thorax 1993; 48: 481-485.

4 Diaz PT, King MA, Pacht ER, et al. The pathophysiology of pulmonary diffusion impairment in human immunodeficiency virus infection. Am J Respir Crit Care Med 1999; 160: 272-277.

5 Mitchell DM, Fleming J, Pinching AJ, et al. Pulmonary function in human immunodeficiency virus infection. A prospective 18-month study of serial lung function in 474 patients. Am Rev Respir Dis 1992; 146: 745-751.

6 Shaw RJ, Roussak C, Forster SM, et al. Lung function abnormalities in patients infected with the human immunodeficiency virus with and without overt pneumonitis. Thorax 1988; 43: 436-440.

7 Gingo MR, George MP, Kessinger CJ, et al. Pulmonary function abnormalities in HIV-infected patients during the current antiretroviral therapy era. Am J Respir Crit Care Med 2010; 182: 790-796.

8 Neas LM, Schwartz J. Pulmonary function levels as predictors of mortality in a national sample of US adults. Am J Epidemiol 1998; 147: 1011-1018.

9 Macintyre N, Crapo RO, Viegi G, et al. Standardisation of the single-breath determination of carbon monoxide uptake in the lung. Eur Respir J 2005; 26: 720-735.

10 Miller MR, Hankinson J, Brusasco V, et al. Standardisation of spirometry. Eur Respir J 2005; 26: 319-338.

11 Neas LM, Schwartz J. The determinants of pulmonary diffusing capacity in a national sample of U.S. adults. Am J Respir Crit Care Med 1996; 153: 656-664.

12 Hankinson JL, Odencrantz JR, Fedan KB. Spirometric reference values from a sample of the general U.S. population. Am J Respir Crit Care Med 1999; 159: 179-187.

13 Leader JK, Zheng B, Rogers RM, et al. Automated lung segmentation in X-ray computed tomography: development and evaluation of a heuristic threshold-based scheme. Acad Radiol 2003; 10: 1224-1236. 
14 Raghu G, Nicholson AG, Lynch D. The classification, natural history and radiological/histological appearance of idiopathic pulmonary fibrosis and the other idiopathic interstitial pneumonias. Eur Respir Rev 2008; 17: 108-115.

15 Kircher BJ, Himelman RB, Schiller NB. Noninvasive estimation of right atrial pressure from the inspiratory collapse of the inferior vena cava. Am J Cardiol 1990; 66: 493-496.

16 Hsue PY, Deeks SG, Farah HH, et al. Role of HIV and human herpesvirus-8 infection in pulmonary arterial hypertension. AIDS 2008; 22: 825-833.

17 Fahy JV, Boushey HA, Lazarus SC, et al. Safety and reproducibility of sputum induction in asthmatic subjects in a multicenter study. Am J Respir Crit Care Med 2001; 163: 1470-1475.

18 Levey AS, Bosch JP, Lewis JB, et al. A more accurate method to estimate glomerular filtration rate from serum creatinine: a new prediction equation. Modification of Diet in Renal Disease Study Group. Ann Intern Med 1999; 130: $461-470$.

19 Tsutamoto T, Wada A, Sakai H, et al. Relationship between renal function and plasma brain natriuretic peptide in patients with heart failure. J Am Coll Cardiol 2006; 47: 582-586.

20 Hosmer DW Jr, Wang CY, Lin IC, et al. A computer program for stepwise logistic regression using maximum likelihood estimation. Comput Programs Biomed 1978; 8: 121-134.

21 Hosmer DW, Hosmer T, Le Cessie S, et al. A comparison of goodness-of-fit tests for the logistic regression model. Stat Med 1997; 16: 965-980.

22 Singh D, Edwards L, Tal-Singer R, et al. Sputum neutrophils as a biomarker in COPD: findings from the ECLIPSE study. Respir Res 2010; 11: 77.

23 Wright JL, Churg A. Current concepts in mechanisms of emphysema. Toxicol Pathol 2007; 35: 111-115.

24 Sitbon O, Lascoux-Combe C, Delfraissy JF, et al. Prevalence of HIV-related pulmonary arterial hypertension in the current antiretroviral therapy era. Am J Respir Crit Care Med 2008; 177: 108-113.

25 Morris A, Gingo MR, George MP, et al. Cardiopulmonary function in individuals with HIV infection in the antiretroviral therapy era. AIDS 2012; 26: 731-740.

26 Glesby MJ, Hoover DR, Shi Q, et al. Glycated haemoglobin in diabetic women with and without HIV infection: data from the Women's Interagency HIV Study. Antivir Ther 2010; 15: 571-577.

27 Marvisi M, Bartolini L, del Borrello P, et al. Pulmonary function in non-insulin-dependent diabetes mellitus. Respiration 2001; 68: 268-272.

28 Park KJ, Bergin CJ, Clausen JL. Quantitation of emphysema with three-dimensional CT densitometry: comparison with two-dimensional analysis, visual emphysema scores, and pulmonary function test results. Radiology 1999; 211: 541-547.

29 Marecki JC, Cool CD, Parr JE, et al. HIV-1 Nef is associated with complex pulmonary vascular lesions in SHIV-nefinfected macaques. Am J Respir Crit Care Med 2006; 174: 437-445.

30 Lipman M, Breen R. Immune reconstitution inflammatory syndrome in HIV. Curr Opin Infect Dis 2006; 19: $20-25$.

31 Gordon C, Gudi K, Krause A, et al. Circulating endothelial microparticles as a measure of early lung destruction in cigarette smokers. Am J Respir Crit Care Med 2011; 184: 224-232.

32 Appay V, Sauce D. Immune activation and inflammation in HIV-1 infection: causes and consequences. J Pathol 2008; 214: 231-241.

33 Appay V, Almeida JR, Sauce D, et al. Accelerated immune senescence and HIV-1 infection. Exp Gerontol 2007; 42: 432-437.

34 Müller KC, Welker L, Paasch K, et al. Lung fibroblasts from patients with emphysema show markers of senescence in vitro. Respir Res 2006; 7: 32.

35 Noureddine H, Gary-Bobo G, Alifano M, et al. Pulmonary artery smooth muscle cell senescence is a pathogenic mechanism for pulmonary hypertension in chronic lung disease. Circ Res 2011; 109: 543-553.

36 Centers for Disease Control and Prevention (CDC). Prevalence of risk behaviors for HIV infection among adults United States, 1997. MMWR Morb Mortal Wkly Rep 2001; 50: 262-265. 